Journal for ImmunoTherapy of Cancer

\title{
Correlation of plasma exosomal microRNAs with the efficacy of immunotherapy in EGFR/ALK wild- type advanced non-small cell lung cancer
}

Xiao-Xiao Peng, ${ }^{1}$ Ruoying Yu, ${ }^{2}$ Xue $\mathrm{Wu},{ }^{2}$ Shu-Yu Wu, ${ }^{3}$ Can $\mathrm{Pi},{ }^{1}$ Zhi-Hong Chen, ${ }^{1}$ Xu-Chao Zhang, ${ }^{1}$ Cun-Yi Gao, ${ }^{3}$ Yang W Shao, ${ }^{3,4}$ Li Liu, ${ }^{5}$ Yi-Long Wu, ${ }^{1}$ Qing Zhou (i) ${ }^{1}$

To cite: Peng X-X, Yu R, Wu X, et al. Correlation of plasma exosomal microRNAs with the efficacy of immunotherapy in EGFR/ALK wild-type advanced non-small cell lung cancer. Journal for ImmunoTherapy of Cancer 2020;8:e000376. doi:10.1136/jitc-2019-000376

- Additional material is published online only. To view please visit the journal online (http://dx.doi.org/10.1136/jitc2019-000376).

$\mathrm{X}-\mathrm{XP}$ and RY contributed equally.

Accepted 15 December 2019

Check for updates

(c) Author(s) (or their employer(s)) 2020. Re-use permitted under CC BY-NC. No commercial re-use. See rights and permissions. Published by BMJ.

For numbered affiliations see end of article.

Correspondence to Professor Qing Zhou; gzzhouqing@126.com

\section{ABSTRACT}

Background Immunotherapy has become an important treatment option for patients with advanced non-small cell lung cancer (NSCLC). At present, none of these existing biomarkers can effectively stratify true responders and there is an urgent need for identifying novel biomarkers. Exosomes derived from the serum of patients with cancer have been proven to be reliable markers for cancer diagnosis. Here, we explored the possibility of using plasma-derived exosomal microRNAs as potential biomarkers for optimal selection of patients with advanced EGFR/ALK negative NSCLC to immunotherapy. Methods From June 2017 to February 2019, 30 patients with advanced EGFR/ALK wild-type (WT) NSCLC who received PD-1/PD-L1 inhibitors were enrolled. The efficacy evaluation was conducted after every three cycles of treatment according to RECIST 1.1. Plasma samples of these patients were collected before the administration of PD-1/PD-L1 inhibitors as baseline, and after every three cycles if the patients achieved partial response (PR) or complete response. Plasma from seven healthy individuals was also collected as normal control. Exosomes were prepared by ultracentrifugation followed by total RNA extraction, and exosome-derived miRNAs were profiled using small RNA next-generation sequencing followed by differential expression analysis.

Results In order to identify biomarker for better response, all five patients who achieved PR and four patients with progressive disease (PD) at efficacy evaluation were included for differential expression analysis. Based on unsupervised hierarchical clustering, exosomal miRNA expression profile was significantly altered in patients with NSCLC compared with normal controls with a total of 155 differentially expressed exosomal miRNAs. Interestingly, hsa-miR320d, hsa-miR-320c, and hsa-miR-320b were identified significantly upregulated in the PD groups compared with the PR group at baseline before the treatment. In addition, we identified that hsa-miR-125b-5p, a T-cell suppressor, showed a trend of increased expression in the PD group at baseline and was significantly downregulated in the post-treatment plasma exosomes compared with pre-treatment samples of the PR patients.
Conclusion Patients with NSCLC represent unique plasma exosomal miRNA profiles. Hsa-miR-320d, hsa-miR-320c, and hsa-miR-320b were identified as potential biomarkers for predicting the efficacy of immunotherapy in advanced NSCLCs. When T-cell suppressor hsa-miR-125b-5p was downregulated during the treatment, the patients may obtain increased T-cell function and respond well to immunotherapy.

\section{BACKGROUND}

With the advent of immune checkpoint blockade therapy, a new therapeutic era has opened for patients with cancer. Immunotherapy has shown promising results in various types of cancer, and immune checkpoint inhibitor (ICI) drugs targeting the PD-1/PD-L1 (programmed cell death protein 1, CD279; programmed deathligand 1, CD274) ${ }^{12}$ and CTLA-4 (cytotoxic T-lymphocyte-associated protein 4 , CD152) ${ }^{3}$ have been approved for treating patients with advanced melanoma, ${ }^{45}$ metastatic renal cell carcinoma, ${ }^{6}$ and advanced non-small cell lung cancer (NSCLC). ${ }^{7}$ ICIs showed a survival advantage in comparison with chemotherapy in first-line and second-line setting of patients with NSCLC; however, overall response rate is around $20 \%$ with a large proportion of patients undergoing disease progression within the first week of treatment. ${ }^{89}$ To spare patients from ineffective therapy, the search for predictive biomarkers to select patients who would preferentially benefit from immunotherapy becomes important.

The expression of PD-L1 on tumor cell membranes has been most widely studied as the predictive biomarker for anti-PD-1/ PD-L1 therapy. ${ }^{10} 11$ However, challenges remain mainly for several reasons. First, the 
use of different PD-L1 antibodies and corresponding cutoffs of its expression level makes it hard to compare and standardize. ${ }^{12}$ Second, the expression of PD-L1 can be dynamic and inducible during the recognition of T-cell antigen which may interfere with treatment response. ${ }^{13}$ Third, the presence of high heterogeneity of PD-L1 expression between primary tumor and metastatic tumor in the same patient limits the robustness of the testing. ${ }^{14}$ Finally, certain patients do not have tumor tissues available for PD-L1 test. High tumor mutational burden, ${ }^{15} 16$ high microsatellite instability, and mismatch repair deficiency ${ }^{17} 18$ are other emerging biomarkers that are significantly associated with response to PD-1/PD-L1 blockade immunotherapy although viable predictive values were observed within different cancer types and clinical trials. High degree of tumor-infiltrating lymphocytes has also been proven to be associated with improved survival in patients with NSCLC and is considered as a potential biomarker for checkpoint inhibitor immunotherapy. ${ }^{19} 20$ However, the establishment of a clinically feasible cutoff is very difficult due to the overlap of baseline T-cell density between patients with or without response. So far, the use of a single biomarker alone has not been sufficient for selection of patients with anti-PD-1/PD-L1 therapy. Further investigations for more robust biomarkers to better stratify the patients are needed.

Exosomes are small membrane vesicles with diameters of $30-100 \mathrm{~nm}$ that can be released by most cell types including cancer cells, which present in various body fluids including plasma, saliva, urine, and malignant effusions. ${ }^{21}$ Exosomes can transfer functional protein, mRNA, and microRNA (miRNA) to neighboring cells, fulfilling its role as the mediator of intercellular communication. ${ }^{22}$ miRNAs are enriched in exosomes and can regulate targeted mRNA translation or stability, and are implicated in the regulation of maturation, proliferation, differentiation, and activation of immune cells. ${ }^{23}{ }^{24}$ The structure of exosomes protects miRNAs from degradation that is suitable for clinical detection.

In patients with cancer, exosomes derived from the serum of patients with cancer have been proven to be reliable markers for cancer diagnosis. ${ }^{25}{ }^{26}$ Cancersecreted exosomes can function as cell-to-cell mediators of metastasis, which prime pre-metastatic niche formation in distant organs ${ }^{27} 28$ and are responsible for engraftment and survival of the metastatic cells. ${ }^{29}$ In this study, we are aiming to explore potential plasma-derived exosomal miRNA signatures as potential biomarkers for the optimal selection of patients with advanced NSCLC to immunotherapy.

\section{METHODS}

\section{Patients and clinical sample collection}

Thirty patients with EGFR/ALK negative lung cancer with available clinical information including age, gender, stage, treatment history, and baseline plasma samples were enrolled in this study in Guangdong Provincial People's
Hospital from June 2017 to February 2019. Peripheral blood was collected from each patient on a regular basis for routine clinical care. Plasma sample was prepared within 2 hours of blood drawn and then stored at $-80^{\circ} \mathrm{C}$. In this study, plasma samples of patients with advanced EGFR/ALK wild-type (WT) NSCLC were collected before the administration of PD-1/PD-L1 inhibitors as baseline. The efficacy evaluation was conducted after three cycles of treatment. For every three cycles, plasma samples were collected from patients until the disease progressed. Patients who achieved partial response (PR) or complete response (CR) were included in this study as responders, compared with patients with progressive disease (PD) on treatment as non-responders. Flow chart for patient selection and exclusion criteria is shown in online supplementary figure 1 . No CR was observed in this patient cohort. In total, five patients who achieved PR and four patients with PD at efficacy evaluation were included in this study. Plasma samples from seven healthy individuals were also collected as normal controls.

Online supplementary table 1 lists the baseline clinicopathological characteristics of all the patients enrolled in this study. formalin-fixed paraffin-embedded(FFPE) slides of the tumor samples were prepared for PD-L1 expression evaluation using immunohistochemistry with the following PD-L1 antibody clones: SP142 or SP263 (Roche, USA), and 28-8 (Abcam, USA). For the nine patients (responders and non-responders) included in this study, seven patients were analyzed using SP263 antibody, while the other two were analyzed with SP142 or 28-8, respectively. Patients were treated with different immunotherapy drugs targeting PD-1/PD-L1. The median follow-up time was 8 months, ranging from 1 month to approximately 21 months. Except one patient with lymphoepithelioma-like carcinoma, five patients were diagnosed with adenocarcinoma, while the other three patients were diagnosed with squamous cell carcinoma. The median age at diagnosis is 55 , ranging from 47 to 68 .

\section{Plasma exosomal RNA isolation and small RNA sequencing} One milliliter of plasma sample was centrifuged at $10,000 \times g$ for $30 \mathrm{~min}$ at $4^{\circ} \mathrm{C}$ to remove any cell debris. The collected supernatant was then subjected for ultra-highspeed centrifugation at $150,000 \times \mathrm{g}$ for $70 \mathrm{~min}$ at $4^{\circ} \mathrm{C}$. The pellet containing exosome was resuspended in $200 \mu \mathrm{L}$ Phosphate buffered saline(PBS) for downstream applications. Total RNA including miRNA was extracted from plasma-derived exosome using miRNeasy Serum/Plasma Kit (QIAGEN) following the manufacturer's instructions. The quantification and size distribution of the extraction were analyzed by Qubit V.4.0 and Agilent Bioanalyzer 2100 (Agilent), respectively. Quantified RNA was subjected for sequencing library preparation using NEBNext Small RNA Library Prep Set for Illumina (NEB Biolabs) following the manufacturer's instructions. Briefly, isolated total RNA was subjected for $3^{\prime}$ and $5^{\prime}$ adaptor ligation, followed by 17 cycles of PCR amplification. PCR products from library preparation were subjected for gel electrophoresis on $6 \%$ 
Novex TBE PAGE gel (Thermo Fisher Scientific) and DNA fragments between 140 and $150 \mathrm{bp}$ were recovered from the gel. Purified small RNA cDNA library was quantified by Qubit V.4.0 and the size distribution was analyzed on Agilent Bioanalyzer 2100, followed by sequencing on Illumina HiSeq 4000 platform.

\section{Characterization of purified exosomes}

For verification of purified exosomes using electron microscopy, purified exosomes suspended in PBS were dropped on copper-coated grids. After staining with $2 \%$ uranyl acetate, grids were dried at room temperature and visualized using a Hitachi H-7650 transmission electron microscope. miRNA-seq data analysis miRNA identification and reads counting in each miRNA were performed using miRDeep2. ${ }^{30}$ After trimming the 3' adaptor sequence, all sequences ranging in length from 18 to $26 \mathrm{nt}$ were recorded in a non-redundant file along with reads count. To identify known miRNAs, the miRNA tags were aligned against miRNA precursor sequences reported in the miRNA database "miRBase" (release 21) using the "quantifier.pl" script within miRDeep2. Differential expression (DE) analysis of miRNA sequence data was performed with the Bioconductor package edgeR. ${ }^{31}$ miRNAs with read counts per million mapped reads $(\mathrm{CPM}) \geq 5$ in at least $20 \%$ of all samples were identified as expressed miRNAs. DE between different groups was evaluated by fitting a negative binomial generalized linear model and then adjusting the $\mathrm{p}$ value for multiple testing using the Benjamini-Hochberg correction with a false discovery rate (FDR) of 0.1 and a minimum $\log _{2}(\mathrm{CPM})$ of 4 .

\section{RESULTS}

\section{Exosomal miRNA expression profiles}

All five patients who achieved PR (responders) and four patients with PD (non-responders) were enrolled in the exosomal miRNA differential expression analysis. Baseline clinicopathological characteristics of patients enrolled are listed in online supplementary table 1 with detailed treatment history of the patients listed in online supplementary table 2. Nine baseline pre-treatment plasma samples and the post-treatment plasma samples from four responders as well as normal control samples from seven healthy individuals were collected for exosome purification and exosomal miRNA profiling. We purified exosomes from plasma by differential centrifugation and verified them as small double-leaflet membrane particles (30-200 nm) by transmission electron microscopy (TEM) (figure 1).

Raw reads of miRNA-seq from all plasma samples were normalized to CPM and 706 miRNAs were retained as expressed miRNAs in plasma exosomes (online supplementary table 3). Using hierarchical clustering, healthy individuals could be distinguished from patients with lung cancer based on expressed miRNAs profile in plasma-derived exosomes (figure 2). We further analyzed

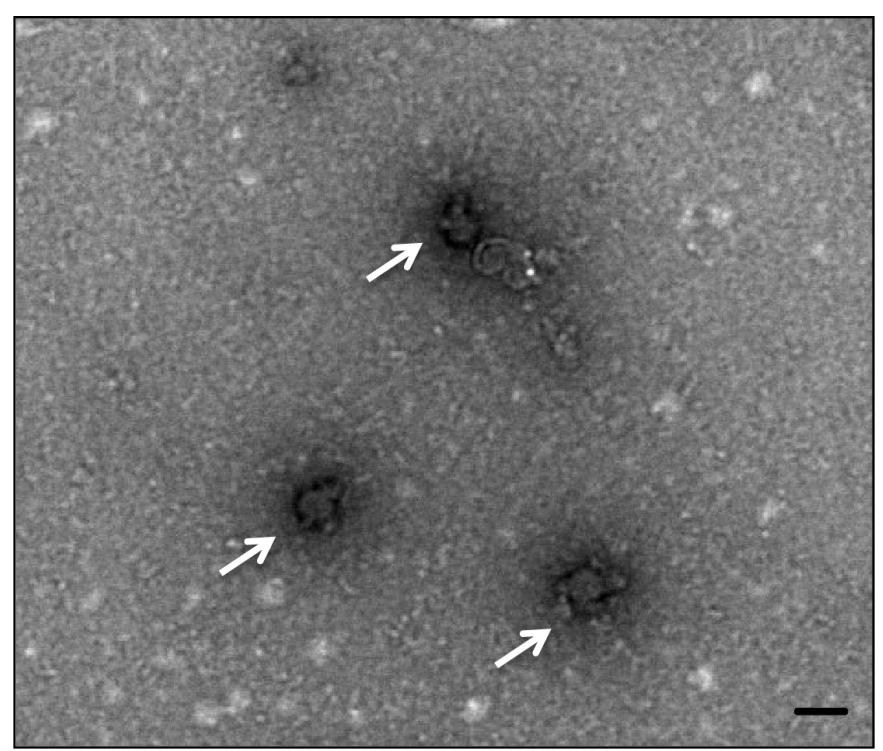

Figure 1 Plasma-derived exosomes. Representative TEM image of purified exosomes from plasma. Arrowheads indicate exosomes. Scale bar, $50 \mathrm{~nm}$.

differentially expressed miRNAs (DE miRNAs) between healthy control samples and patient samples. As shown in online supplementary table 4, $155 \mathrm{DE}$ miRNAs were identified and the top 10 upregulated and top 10 downregulated miRNAs are listed in online supplementary table 4a. Some of these top regulated miRNAs were highly related to immune pathways. Among them, hsa-miR-146a has been well studied in autoimmune disease pathogenesis and is related to NF-KB pathway involved in immune response. $^{32}$ hsa-miR-221 and hsa-miR-224 have been
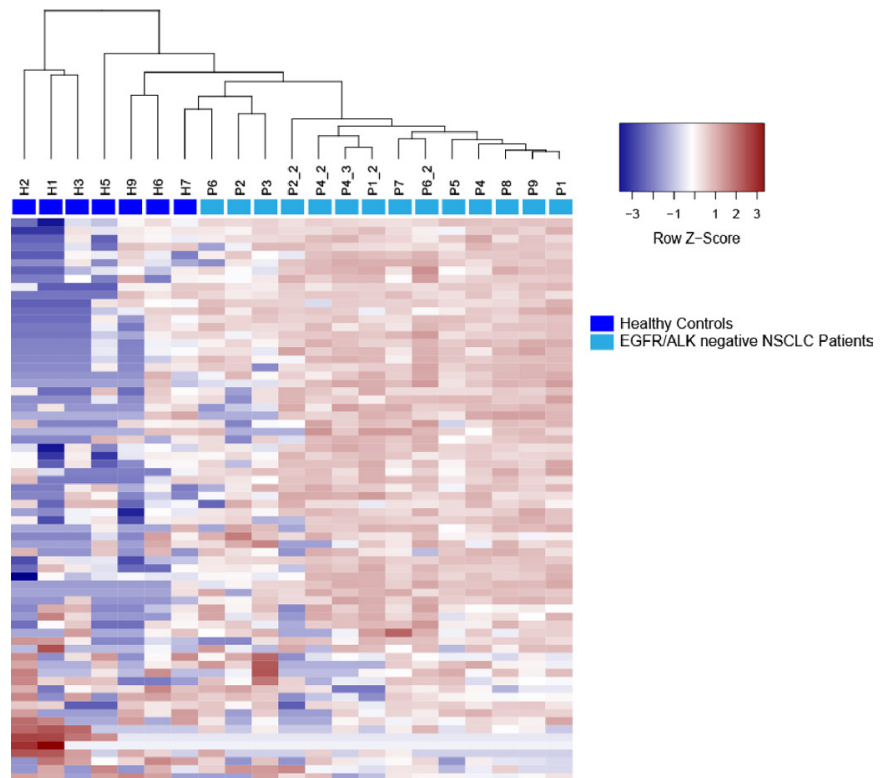

Figure 2 Plasma-derived exosomal miRNAs separated healthy control samples from EGFR/ALK negative non-small cell lung cancer (NSCLC) patient samples. Unsupervised hierarchical clustering of the miRNA expression profiles from plasma exosome clearly separated healthy control samples from $E G F R / A L K$ negative lung cancer patient group. 
reported to be related to anti-TNF treatment in inflammatory bowel disease. ${ }^{33}$ hsa-miR-224 has been proven to induce cell proliferation by target Smad4 through SMAD/TGF- $\beta$ pathway. ${ }^{34}$ Meanwhile, three of the top regulated miRNAs which were downregulated in patients with lung cancer have been reported as tumor suppressors in different cancer types, including hsa-miR-144, ${ }^{35-37}$ hsa-miR-363-3p, ${ }^{38}$ and hsa-miR-150. ${ }^{39} 40$

Kyoto Encyclopedia of Genes and Genomes(KEGG) pathway analysis was performed with 155 differentially expressed exosomal miRNAs using DIANA tool (online supplementary table 4b). Cancer-related pathways were significantly enriched in lung cancer patient group including TGF- $\beta$, MAPK, Hippo, and Ras signaling pathways. Immune-related pathways including TNF signaling pathway, B-cell and T-cell receptor signaling pathways were also enriched in the patient group. In conclusion, plasma-derived exosomes' miRNA expression profile was altered in patients with $E G F R / A L K$ negative lung cancer and differently expressed miRNAs were enriched in cancer-related and immune-related pathways compared with healthy control.

\section{Different exosomal miRNA expression profiles between PR and $\mathrm{PD}$ patients}

To identify the potential predictors of anti-PD-1 therapy in patients with lung cancer, we divided patient samples into PR group and PD group. Then we compared the miRNA profile between PR group and PD group using exact test. With cut-off at $\log \mathrm{CPM}>4, \mathrm{p}<0.05, \mathrm{FDR} \leq 0.1$, hsa-miR-320d was considered as the most significant DE miRNA followed by hsa-miR-642a-3p, hsa-miR-320c, and hsa-miR-320b with FDR around 0.1 (table 1 ).

Next, we examined the miRNA expression level of hsamiR-642a-3p, hsa-miR-320c, hsa-miR-320b, and hsa-miR642a-3p in overall patient cohort and healthy population (H). We separated all the samples from patients into

Table 1 Identification of plasma-derived exosomal miRNAs as potential predictors for anti-PD-1 therapy in patients with lung cancer

\begin{tabular}{|lcccl|}
\hline $\begin{array}{l}\text { Top 10 DE } \\
\text { miRNAs }\end{array}$ & logFC & logCPM & P value & FDR \\
\hline hsa-miR-320d & 2.478246 & 7.953041 & $8.56 E-05$ & 0.050411 \\
\hline hsa-miR-642a-3p & 4.429584 & 2.612111 & 0.000513 & 0.102554 \\
\hline hsa-miR-320c & 1.919471 & 9.497452 & 0.000522 & 0.102554 \\
\hline hsa-miR-320b & 1.678455 & 10.70763 & 0.001134 & 0.167017 \\
\hline hsa-miR-200a-3p & 2.024362 & 5.74316 & 0.003509 & 0.370143 \\
\hline hsa-miR-365a-3p & 4.031761 & 1.961358 & 0.004822 & 0.370143 \\
\hline hsa-miR-365b-3p & 4.032808 & 1.961358 & 0.004948 & 0.370143 \\
\hline hsa-miR-4662a-5p & -2.83216 & 3.325355 & 0.005027 & 0.370143 \\
\hline hsa-miR-10a-3p & 2.697804 & 4.247801 & 0.008292 & 0.542682 \\
\hline hsa-miR-6511b-3p & -2.55458 & 3.294422 & 0.011099 & 0.653718 \\
\hline
\end{tabular}

Differentially expressed (DE) miRNAs between PD and PR patients were analyzed by exact test (logCPM $>4, p<0.05, F D R \leq 0.1)$.

CPM, counts per million; FC, Fold change; FDR, false discovery rate; $P D$, progressive disease; PR, partial response. three groups: PR pre-treatment samples (PR-pre), PD pre-treatment samples (PD-pre), and PR post-treatment samples (PR-post). As shown in figure 2, hsa-miR-320d, hsa-miR-320c, and hsa-miR-320b belonged to hsa-miR320 family and all showed significant difference in mRNA expression level between PD-pre group and PR-pre group. Moreover, compared with healthy control and PR group (PR-pre and PR-post), hsa-miR-320d, hsa-miR-320c, and hsa-miR-320b showed upregulation in PD-pre group (figure 3). hsa-miR-643a-3p was eliminated due to its low expression level and no significant difference in miRNA expression level among these three groups (figure 3D). In conclusion, high level of hsa-miR-320d, hsa-miR-320c, and hsa-miR-320b might correlate with unfavorable response to anti-PD-1 treatment and hsa-miR-320d, hsamiR-320c, and hsa-miR-320b might be potential predictors of anti-PD-1 therapy.

\section{Identification of plasma-derived exosomal miRNAs suppressed by anti-PD-1 therapy in patients with lung cancer} Paired pre-treatment and post-treatment plasma samples were collected from the four patients who achieved PR on anti-PD-1 therapy (online supplementary table 1). To identify potential exosomal miRNAs influenced by antiPD-1 therapy during the treatment course, we compared the exosomal miRNA profiles before (PR-pre) and after (PR-post) anti-PD-1 treatment. With cut-off at $\log \mathrm{CPM}>4$, $\mathrm{p}<0.05$, FDR $\leq 0.1$, only hsa-miR-125b-5p was differentially expressed between PR-pre and PR-post samples (table 2 and figure $4 \mathrm{~A}$ ), which was dramatically downregulated in the PR-post samples and suggested that the level of exosomal hsa-miR-125b-5p might be used as a potential indicator to monitor the efficacy of anti-PD-1 treatment. Interestingly, plasma samples from the PD group at baseline exhibited a trend of increased level of hsamiR125b-5p compared with PR-pre group and healthy controls (figure $4 \mathrm{~A}$ ).

This study has demonstrated that hsa-miR-125b-5p could induce $\gamma \delta$ T-cell apoptosis and downregulate $\gamma \delta$ T-cell activation and cytotoxicity to tumor cells by decreasing degranulation and inhibiting the secretion of IFN- $\gamma$ and TNF- $\mathrm{a}^{41}$ (figure 4B). An increased exosomal hsa-miR-125b-5p level in PD group at baseline might indicate impaired $\gamma \delta$ T-cell function and cytotoxicity to tumor cells that could confer resistance to immunotherapy. On the other hand, a significantly reduced exosomal hsa-miR125b-5p level could indicate the activation of $\mathrm{T}$ cells and cytotoxicity to tumor cells in response to immunotherapy.

\section{DISCUSSION}

Here, we identified three miRNAs from hsa-miR-320 family as potential predictors and one potential target hsa-miR-125b-5p for anti-PD-1 treatment. The three miRNAs, hsa-miR-320d, hsa-miR-320c, and hsa-miR-320b, all exhibited upregulation in PD group compared with PR group and correlated with unfavorable response to anti-PD-1 treatment. hsa-miR-125b-5p was downregulated 

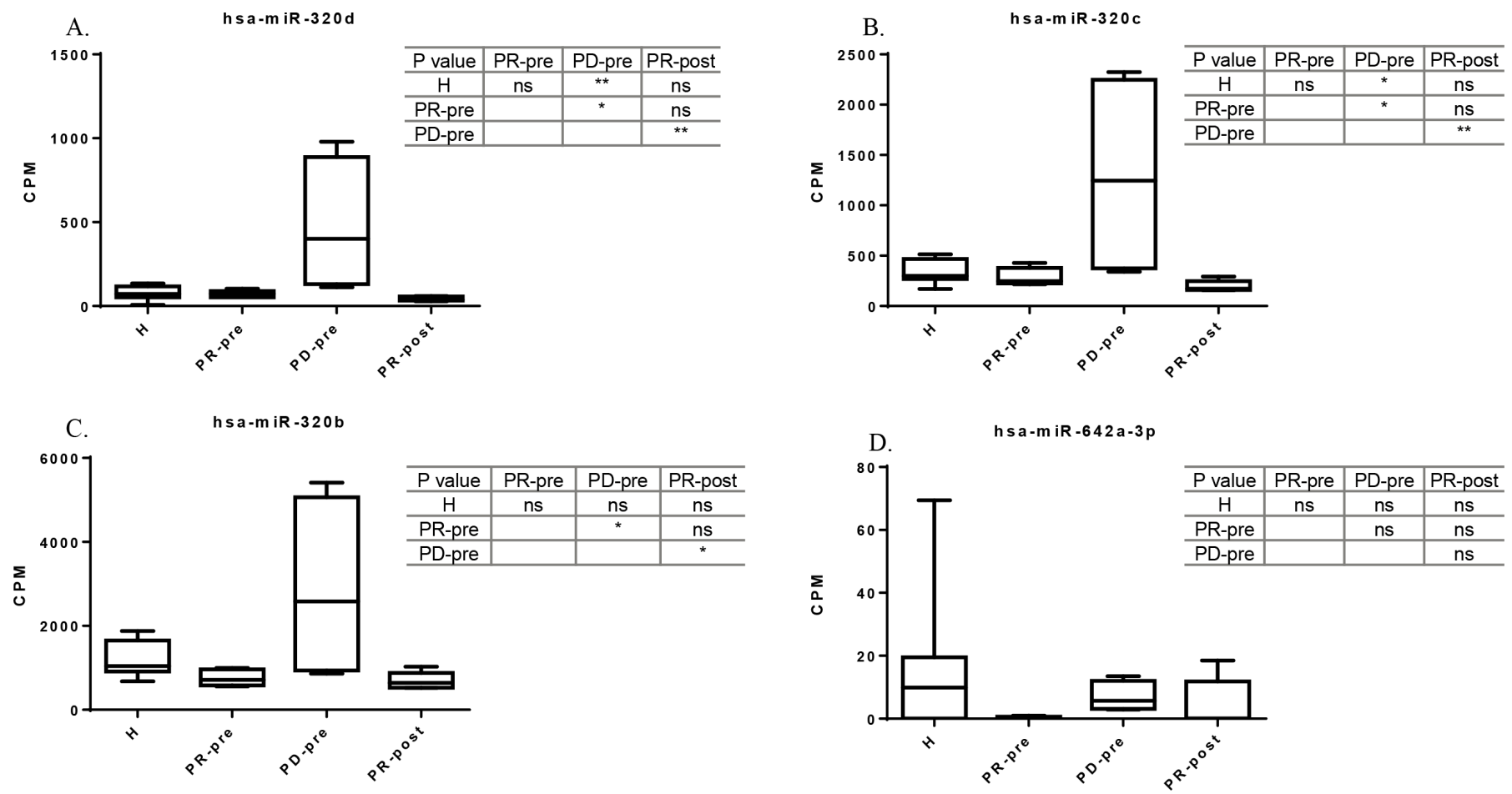

Figure 3 Three members from miR320 family were only upregulated in PD pre-treatment samples. Box plot showing the individual CPM of hsa-miR-320d (A), hsa-miR-320c (B), hsa-miR-320d (C), and hsa-miR-642a-3p (D) in each sample group. P value was calculated using ANOVA. CPM, counts per million; $\mathrm{H}$, healthy control group; ns, not significant; PD-pre, progressive disease group pre-treatment samples; PR-post, partial response group post-treatment samples; PR-pre, partial response group pre-treatment samples. ${ }^{*} \mathrm{p}<0.05 ;{ }^{* \star} \mathrm{p}<0.01$.

in patients with response to anti-PD-1 treatment which might be a target for anti-PD-1 therapy. Meanwhile, hsa-miR-125b-5p exhibited a trend of increase in PD group compared with PR group and healthy individual group. Based on the results, patients with low level of hsa-miR-320d, hsa-miR-320c, hsa-miR-320b, and hsamiR-125b-5p might be better candidates for anti-PD-1 treatment. During the anti-PD-1 treatment, continuous decrease in the level of hsa-miR-125b-5p may be taken into consideration as a sign of better outcome and longer

Table 2 Identification of plasma-derived exosomal miRNAs as potential targets for anti-PD-1 therapy in patients with lung cancer

\begin{tabular}{|lllll|}
\hline Top 10 miRNAs & logFC & logCPM & P value & FDR \\
\hline hsa-miR-125b-5p & -2.12201 & 8.80358 & $7.69 E-05$ & 0.053356 \\
\hline hsa-miR-1246 & -2.21223 & 6.7894305 & 0.001474 & 0.511533 \\
\hline hsa-miR-1290 & -2.40124 & 5.7791914 & 0.002272 & 0.525701 \\
\hline hsa-miR-144-5p & -1.5785 & 8.8879329 & 0.003899 & 0.676561 \\
\hline hsa-miR-4676-3p & -5.39321 & 1.6555446 & 0.006068 & 0.719744 \\
\hline hsa-miR-4433b-3p & 1.962046 & 9.7763291 & 0.006392 & 0.719744 \\
\hline hsa-miR-744-5p & 0.985601 & 12.645567 & 0.007612 & 0.719744 \\
\hline hsa-miR-7976 & -2.93799 & 3.7599466 & 0.009889 & 0.719744 \\
\hline hsa-miR-877-5p & 1.539099 & 7.1799127 & 0.009925 & 0.719744 \\
\hline hsa-miR-10b-3p & -3.14894 & 4.1456507 & 0.010937 & 0.719744 \\
\hline
\end{tabular}

Differentially expressed miRNAs between PR-pre and PR-post samples were analyzed by exact test $(\log C P M>4, p<0.05, F D R \leq 0.1)$.

CPM, counts per million; FC, Fold change; FDR, false discovery rate. progression-free survival(PFS). Especially, in the patient with delayed clinical responses or pseudoprogression, the level of hsa-miR-125b-5p might also be used as a potential prognostic marker to indicate whether the patient would benefit from anti-PD-1 treatment.

The employment of plasma-derived exosomal miRNAs in anti-PD-1 therapy holds some advantages in clinical circumstances. First, the plasma-derived exosomes detected in patients are mainly released from tumor cells which can be a more accurate and dynamic reflection of the status and function of tumor cells, rather than tumor tissue. Second, miRNAs in exosomes are more stable compared with miRNAs in blood due to the double-membrane structure. Furthermore, the detection of exosomal miRNAs only requires patient blood sample which is able to overcome spatial heterogeneity of tissue samples and timely monitor tumor progression during the whole treatment course. In future application, we can go further to simplify this method by using qPCR to monitor miRNA biomarkers in plasma-derived exosomes.

The miR320 family which consists of five members (hsamiR-320a, hsa-miR-320b, hsa-miR-320c, hsa-miR-320d, hsa-miR-320e) is conserved but only exists in vertebrates from Xenopus to humans. hsa-miR-320d and hsa-miR-320e only present in primates and humans. ${ }^{42}$ High expression of hsa-miR-320 has been reported to be associated with negative prognosis and high risk of metastasis in ovarian cancer. ${ }^{43}$ Overexpression of hsa-miR-320 promotes B-cell proliferation through suppressing Phosphatase and tensin 


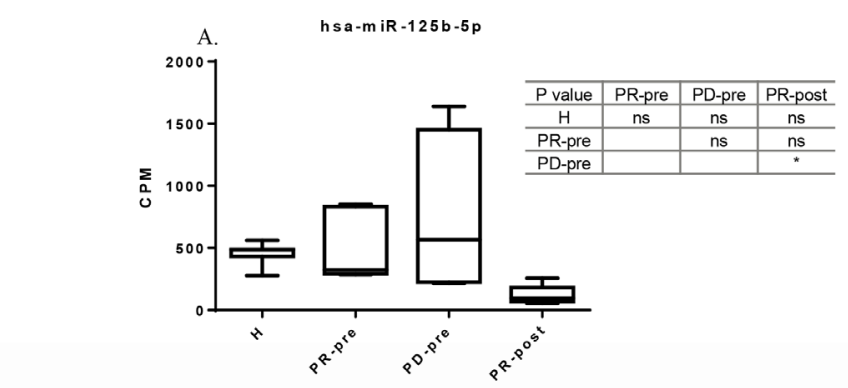

B.Chromosome11

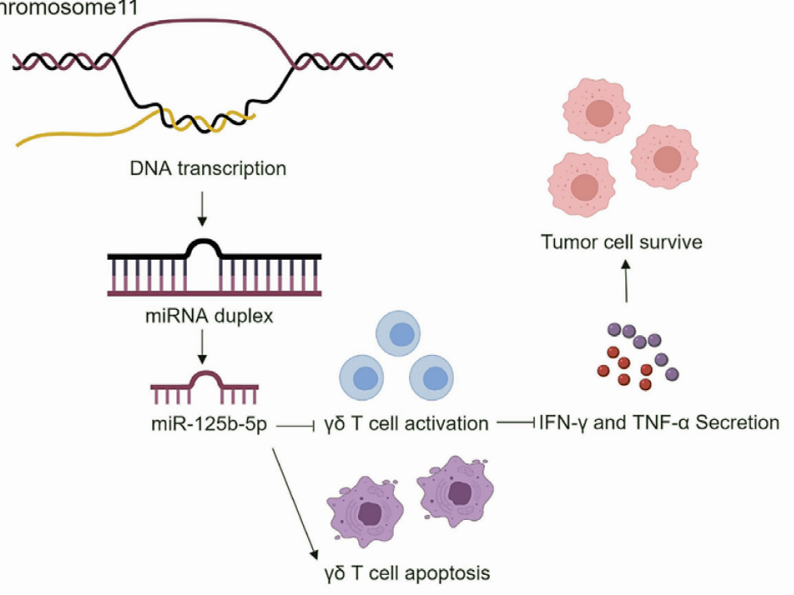

$\longrightarrow$ : inhibiting downstream event $\longrightarrow$ : affected downstream event

Figure 4 hsa-miR-125b-5p was downregulated after anti-PD-1 treatment. (A) Box plot showing the individual CPM of hsa-miR-125b-5p in each sample group. $P$ value was calculated using ANOVA. CPM, counts per million; $\mathrm{H}$, healthy control group; ns, not significant; PD-pre, progressive disease group pre-treatment samples; PR-post, partial response group post-treatment samples; PR-pre, partial response group pre-treatment samples. ${ }^{*} p<0.05 ;{ }^{* *} p<0.01$. (B) Schematic diagram of potential regulation mechanism of hsa-miR-125b-5p on $\gamma \delta$ T cell.

homolog(PTEN) expression and promotes cyclin D1 expression. ${ }^{44}$ Our data also confirmed that high expression of hsa-miR-320d, hsa-miR-320c, and hsa-miR-320b has a negative prognosis and associated with bad response to anti-PD-1 treatment in patients with NSCLC. The observation of downregulated hsa-miR-125b-5p in PR-pre group also supported the role of hsa-miR-125b-5p as a T-cell suppressor in another study. We could further validate these observations by detecting the level of hsa-miR-320d, hsa-miR-320c, hsa-miR-320b, and hsa-miR-125b-5p in the PD group after anti-PD-1 treatment. However, plasma samples from PD group after anti-PD-1 treatment were not available for analysis in this study because the evaluation was stopped after the patient showed PD. Future studies need to be carried out for further investigation and validation.

Unfortunately, we did not compare these potential biomarkers with other markers to explore their prediction accuracy. The other limitation of our study is that the cohort we used is small. There are other clinicopathological factors such as smoking status ${ }^{45}$ and treatment history that may also contribute to the upregulation or downregulation of exosomal miRNAs. Therefore, these four miRNAs need to be tested in other cohorts to validate their roles as potential immunotherapy biomarkers.

In conclusion, we identified four plasma-derived exosomal miRNAs as potential biomarkers of anti-PD-1 therapy for patients with NSCLC which can be indicators for non-invasive longitudinal liquid biopsy to ruling in or ruling out patients undergo immunotherapy. Large cohort validation and functional study of these potential biomarkers are required before it can be applied to clinical use.

\section{CONCLUSIONS}

The exosomal miRNA profile of patients with NSCLC differs from healthy individuals. We identified three miRNAs from hsa-miR-320 family as potential predictors and one potential target hsa-miR-125b-5p for anti-PD-1 treatment in plasma exosomes of patients with NSCLC. Plasma-derived exosomal miRNAs may be potential biomarkers for antiPD-1 therapy in EGFR/ALK wild-type advanced NSCLC.

\section{Author affiliations \\ ${ }^{1}$ Guangdong Lung Cancer Institute, Guangdong Provincial Key Laboratory of Translational Medicine in Lung Cancer, Guangdong Provincial People's Hospital and Guangdong Academy of Medical Sciences, Guangzhou, China \\ ${ }^{2}$ Translational Medicine Research Institute, Geneseeq Technology Inc, Toronto, Ontario, Canada \\ ${ }^{3}$ Department of Research and Development, Nanjing Geneseeq Technology Inc, Nanjing, China \\ ${ }^{4}$ School of Public Health, Nanjing Medical University, Nanjing, Jiangsu, China ${ }^{5}$ Cancer Center, Union Hospital, Tongji Medical College, Huazhong University of Science and Technology, Wuhan, China}

Contributors X-XP collected and interpreted the patient data, and wrote the manuscript. R- YY analyzed data and wrote the manuscript. XW, S-YW, CP, Z-HC, X$\mathrm{CZ}$, YWS, LL, and Y-LW reviewed and edited the manuscript. C-YG was responsible for TEM data of the manuscript. QZ was responsible for conceptualization, funding, and methodology of the manuscript. All authors read and approved the final manuscript.

Funding The study was supported by National Key R\&D Program of China (grant no. 2016YFC1303800 to QZ), National Natural Science Foundation of China (no. 81871891 to QZ), and High-level Hospital Construction Project (grant no. DFJH201810 to QZ).

Competing interests XW and R-YY are employees of Geneseeq Technology Inc. YWS, S-YW, and C-YG are shareholders or employees of Nanjing Geneseeq Technology Inc. Y-LW has received speaker fees from AstraZeneca, Roche, Eli Lilly, Pfizer, and Sanofi. QZ declares speaker fees from AstraZeneca and Roche.

Patient consent for publication Obtained.

Ethics approval The study was approved by the Ethics Committee at Guangdong Provincial People's Hospital.

Provenance and peer review Not commissioned; externally peer reviewed. Data availability statement Data are available on reasonable request.

Open access This is an open access article distributed in accordance with the Creative Commons Attribution Non Commercial (CC BY-NC 4.0) license, which permits others to distribute, remix, adapt, build upon this work non-commercially, and license their derivative works on different terms, provided the original work is properly cited, appropriate credit is given, any changes made indicated, and the use is non-commercial. See http://creativecommons.org/licenses/by-nc/4.0/.

ORCID iD

Qing Zhou http://orcid.org/0000-0002-0478-176X 


\section{REFERENCES}

1 Topalian SL, Hodi FS, Brahmer JR, et al. Safety, activity, and immune correlates of anti-PD-1 antibody in cancer. N Engl J Med 2012;366:2443-54

2 Brahmer JR, Tykodi SS, Chow LQM, et al. Safety and activity of anti-PD-L1 antibody in patients with advanced cancer. $N$ Engl $J$ Med 2012;366:2455-65.

3 Hodi FS, O'Day SJ, McDermott DF, et al. Improved survival with ipilimumab in patients with metastatic melanoma. $N$ Engl J Med 2010;363:711-23.

4 Topalian SL, Sznol M, McDermott DF, et al. Survival, durable tumor remission, and long-term safety in patients with advanced melanoma receiving nivolumab. J Clin Oncol 2014;32:1020-30.

5 Hamid O, Robert C, Ribas A, et al. Antitumour activity of pembrolizumab in advanced mucosal melanoma: a post-hoc analysis of KEYNOTE-001, 002, 006. Br J Cancer 2018;119:670-4.

6 Hammers HJ, Plimack ER, Infante JR, et al. Safety and efficacy of nivolumab in combination with ipilimumab in metastatic renal cell carcinoma: The CheckMate 016 Study. J Clin Oncol 2017;35:3851-8.

7 Reck M, Rodríguez-Abreu D, Robinson AG, et al. Pembrolizumab versus chemotherapy for PD-L1-positive non-small-cell lung cancer. N Engl J Med 2016;375:1823-33.

8 Rittmeyer A, Barlesi F, Waterkamp D, et al. Atezolizumab versus docetaxel in patients with previously treated non-small-cell lung cancer (OAK): a phase 3, open-label, multicentre randomised controlled trial. Lancet 2017;389:255-65.

$9 \mathrm{Kim}$ Y-H. Durvalumab after chemoradiotherapy in stage III non-smallcell lung cancer. N Engl J Med 2019;380:989-90.

10 Kluger HM, Zito CR, Turcu G, et al. PD-L1 studies across tumor types, its differential expression and predictive value in patients treated with immune checkpoint inhibitors. Clin Cancer Res 2017;23:4270-9.

11 Dong Z-Y, Zhong W-Z, Zhang X-C, et al. Potential predictive value of TP53 and KRAS mutation status for response to PD-1 blockade immunotherapy in lung adenocarcinoma. Clin Cancer Res 2017;23:3012-24.

12 Hansen AR, Siu LL. PD-L1 testing in cancer: challenges in companion diagnostic development. JAMA Oncol 2016;2:15-16.

13 Sacher AG, Gandhi L. Biomarkers for the clinical use of PD-1/PDL1 inhibitors in non-small-cell lung cancer: a review. JAMA Oncol 2016;2:1217-22.

14 McLaughlin J, Han G, Schalper KA, et al. Quantitative assessment of the heterogeneity of PD-L1 expression in non-small-cell lung cancer. JAMA Oncol 2016;2:46-54.

15 Schumacher TN, Schreiber RD. Neoantigens in cancer immunotherapy. Science 2015;348:69-74.

16 Rizvi NA, Hellmann MD, Snyder A, et al. Cancer immunology. Mutational landscape determines sensitivity to PD-1 blockade in non-small cell lung cancer. Science 2015;348:124-8.

17 Le DT, Durham JN, Smith KN, et al. Mismatch repair deficiency predicts response of solid tumors to PD-1 blockade. Science 2017;357:409-13.

18 Prasad V, Kaestner V, Mailankody S. Cancer drugs approved based on biomarkers and not tumor type-FDA approval of pembrolizumab for mismatch repair-deficient solid cancers. JAMA Oncol 2018;4:157-8.

19 Bremnes RM, Busund L-T, Kilvær TL, et al. The role of tumorinfiltrating lymphocytes in development, progression, and prognosis of non-small cell lung cancer. J Thorac Oncol 2016;11:789-800.

20 Tokito T, Azuma K, Kawahara A, et al. Predictive relevance of PD-L1 expression combined with CD8+ TIL density in stage III non-small cell lung cancer patients receiving concurrent chemoradiotherapy. Eur J Cancer 2016:55:7-14.

21 Lässer C, Alikhani VS, Ekström K, et al. Human saliva, plasma and breast milk exosomes contain RNA: uptake by macrophages. $J$ Transl Med 2011;9:9.

22 Li J, Liu K, Liu Y, et al. Exosomes mediate the cell-to-cell transmission of IFN- $\alpha$-induced antiviral activity. Nat Immunol 2013;14:793-803.
23 Baltimore D, Boldin MP, O'Connell RM, et al. MicroRNAs: new regulators of immune cell development and function. Nat Immunol 2008;9:839-45

24 Xiao C, Rajewsky K. MicroRNA control in the immune system: basic principles. Cell 2009;136:26-36.

25 Melo SA, Luecke LB, Kahlert C, et al. Glypican-1 identifies cancer exosomes and detects early pancreatic cancer. Nature 2015;523:177-82.

26 Huang X, Yuan T, Liang M, et al. Exosomal miR-1290 and miR-375 as prognostic markers in castration-resistant prostate cancer. Eur Urol 2015;67:33-41.

27 Becker A, Thakur BK, Weiss JM, et al. Extracellular vesicles in cancer: cell-to-cell mediators of metastasis. Cancer Cell 2016;30:836-48.

28 Costa-Silva B, Aiello NM, Ocean AJ, et al. Pancreatic cancer exosomes initiate pre-metastatic niche formation in the liver. Nat Cell Biol 2015;17:816-26.

29 Hoshino A, Costa-Silva B, Shen T-L, et al. Tumour exosome integrins determine organotropic metastasis. Nature 2015;527:329-35.

30 Friedländer MR, Mackowiak SD, Li N, et al. miRDeep2 accurately identifies known and hundreds of novel microRNA genes in seven animal clades. Nucleic Acids Res 2012;40:37-52.

31 Robinson MD, McCarthy DJ, Smyth GK. edgeR: a bioconductor package for differential expression analysis of digital gene expression data. Bioinformatics 2010;26:139-40.

32 Taganov KD, Boldin MP, Chang K-J, et al. NF-kappaB-dependent induction of microRNA miR-146, an inhibitor targeted to signaling proteins of innate immune responses. Proc Natl Acad Sci U S A 2006;103:12481-6.

33 Fujioka S, Nakamichi I, Esaki M, et al. Serum microRNA levels in patients with Crohn's disease during induction therapy by infliximab. J Gastroenterol Hepatol 2014;29:1207-14.

34 Yao G, Yin M, Lian J, et al. MicroRNA-224 is involved in transforming growth factor-beta-mediated mouse granulosa cell proliferation and granulosa cell function by targeting Smad4. Mol Endocrinol 2010;24:540-51.

35 Ren K, Liu Q-Q, An Z-F, et al. MiR-144 functions as tumor suppressor by targeting PIM1 in gastric cancer. Eur Rev Med Pharmacol Sci 2017;21:3028-37.

36 Cheng Z-X, Song Y-X, Wang Z-Y, et al. miR-144-3p serves as a tumor suppressor by targeting FZD7 and predicts the prognosis of human glioblastoma. Eur Rev Med Pharmacol Sci 2017;21:4079-86.

37 Liu F, Chen N, Xiao R, et al. miR-144-3p serves as a tumor suppressor for renal cell carcinoma and inhibits its invasion and metastasis by targeting MAP3K8. Biochem Biophys Res Commun 2016;480:87-93.

38 Song B, Yan J, Liu C, et al. Tumor suppressor role of miR-363-3p in gastric cancer. Med Sci Monit 2015;21:4074-80.

39 Podshivalova K, Wang EA, Hart T, et al. Expression of the miR-150 tumor suppressor is restored by and synergizes with rapamycin in a human leukemia T-cell line. Leuk Res 2018;74:1-9.

40 Leoncini PP, Bertaina A, Papaioannou D, et al. MicroRNA fingerprints in juvenile myelomonocytic leukemia (JMML) identified miR-150-5p as a tumor suppressor and potential target for treatment. Oncotarget 2016;7:55395-408

41 Zhu Y, Zhang S, Li Z, et al. miR-125b-5p and miR-99a-5p downregulate human $\gamma \delta$ T-cell activation and cytotoxicity. Cell $\mathrm{Mol}$ Immunol 2019;16:112-25.

42 Lieb V, Weigelt K, Scheinost L, et al. Serum levels of miR-320 family members are associated with clinical parameters and diagnosis in prostate cancer patients. Oncotarget 2018;9:10402-16.

43 Wang W, Yang J, Xiang Y-Y, et al. Overexpression of Hsa-miR-320 is associated with invasion and metastasis of ovarian cancer. $J \mathrm{Ce} / \mathrm{l}$ Biochem 2017;118:3654-61

44 Li C, Shi J, Zhao Y. MiR-320 promotes B cell proliferation and the production of aberrant glycosylated lgA1 in IgA nephropathy. $J$ Cell Biochem 2018;119:4607-14.

45 Kaur G, Begum R, Thota S, et al. A systematic review of smokingrelated epigenetic alterations. Arch Toxicol 2019;93:2715-40. 
Correction: Correlation of plasma exosomal microRNAs with the efficacy of immunotherapy in EGFR / ALK wild-type advanced non-small cell lung cancer

Peng X, Yu R, Wu X, et al. Correlation of plasma exosomal microRNAs with the efficacy of immunotherapy in EGFR / ALK wild-type advanced non-small cell lung cancer. $J$ Immunother Cancer 2020;8:e00376. doi: 10.1136/jitc-2019-000376

Since the online publication of this article, the authors have noticed that the author name Ruo-Ying Yu was spelt incorrectly. The correct spelling is Ruoying yu.

Open access This is an open access article distributed in accordance with the Creative Commons Attribution Non Commercial (CC BY-NC 4.0) license, which permits others to distribute, remix, adapt, build upon this work non-commercially, and license their derivative works on different terms, provided the original work is properly cited, appropriate credit is given, any changes made indicated, and the use is non-commercial. See http://creativecommons.org/licenses/by-nc/4.0/.

C Author(s) (or their employer(s)) 2020. Re-use permitted under CC BY-NC. No commercial re-use. See rights and permissions. Published by BMJ.

J Immunother Cancer 2020;8:e000376corr1. doi:10.1136/jitc-2019-000376corr1

A) Check for updates 